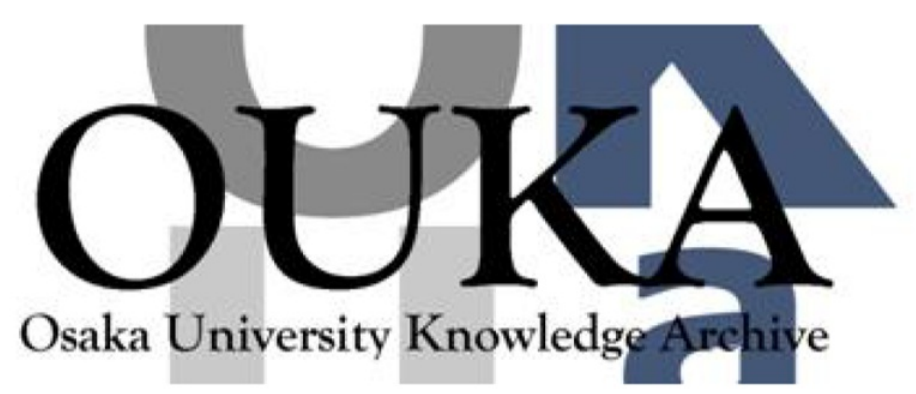

\begin{tabular}{|c|l|}
\hline Title & $\begin{array}{l}\text { Adhesive bond imaging by noncontact } \\
\text { measurements with single-sided access }\end{array}$ \\
\hline Author(s) & Nakao, Shogo; Hayashi, Takahiro \\
\hline Citation & $\begin{array}{l}\text { Journal of Nondestructive Evaluation, } \\
\text { Diagnost ics and Prognost ics of Engineering } \\
\text { Systems. 1(2) p. 021009 }\end{array}$ \\
\hline Issue Date & $2018-02-23$ \\
\hline oaire:version & AM \\
\hline URL & https://hdl. handle. net/11094/85208 \\
\hline rights & $\begin{array}{l}\text { O 2018 ASME. This manuscript version is made } \\
\text { avai lable under the Creative Commons } \\
\text { Attribution 4.0 International License. }\end{array}$ \\
\hline Note & \multicolumn{2}{|c|}{} \\
\hline
\end{tabular}

Osaka University Knowledge Archive : OUKA

https://ir. Library. osaka-u. ac. jp/

0saka University 


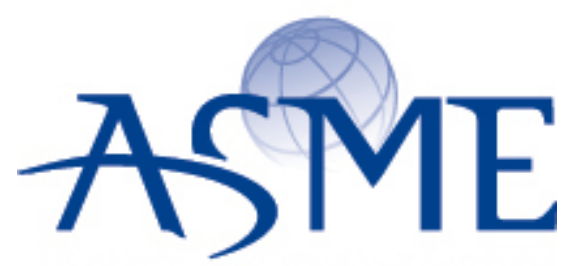

\section{American Society of Mechanical Engineers}

SETTING THE STANDARD

ASME Accepted Manuscript Repository

Institutional Repository Cover Sheet

ASME Paper Title: Adhesive Bond Imaging by Noncontact Measurements With Single-Sided Access

Authors: $\quad$ Shogo Nakao, Takahiro Hayashi

ASME Journal Title: Journal of Nondestructive Evaluation, Diagnostics and Prognostics of Engineering Systems

Volume/Issue _ Volume 1/Issue 2 Date of Publication (VOR* Online) February 23, 2018

ASME Digital Collection URL: https://asmedigitalcollection.asme.org/nondestructive/article/1/2/021009/375442/ Adhesive-Bond-Imaging-by-Noncontact-Measurements?searchresult=1

DOI: $\quad 10.1115 / 1.4039229$

*VOR (version of record) 


\title{
Adhesive bond imaging by noncontact measurements with single-sided access
}

\author{
Shogo Nakao \\ Graduate School of Engineering, Kyoto University \\ Kyotodaigaku-katsura, Nishikyo-ku, Kyoto 615-8540, Japan \\ nakao.shogo.24w@st.kyoto-u.ac.jp \\ Takahiro Hayashi ${ }^{1}$ \\ Graduate School of Engineering, Kyoto University \\ Kyotodaigaku-katsura, Nishikyo-ku, Kyoto 615-8540, Japan \\ hayashi@kuaero.kyoto-u.ac.jp
}

\begin{abstract}
Adhesive bonding, an effective joining technique for plate-like structures in aircraft and automobiles, requires post-bond inspection preferably with noncontact and single-sided access. The present study discusses the application of an imaging technique with a scanning laser source (SLS) to evaluate adhesive bonds in a plate-like structure. When a laser doppler vibrometer is used as a receiver, the SLS technique realizes noncontact measurements with single-sided access. The imaging experiments that used narrowband burst waves and broadband chirp waves indicated that the imaging technique is appropriately applied to adhesive bonds and that the use of broadband chirp waves provides clearer images and reduces spurious images due to resonance. Furthermore, images of adhesive bonds were clearly obtained for a complex plate-structure that consisted of a top-hat section and a flat plate, and this demonstrates that the imaging technique can be widely applied to evaluate various adhesive bonds.
\end{abstract}

\footnotetext{
${ }^{1}$ Corresponding author : T. Hayashi
} 
Journal of Nondestructive Evaluation, Diagnostics and Prognostics of Engineering Systems

\section{INTRODUCTION}

In aircraft and automobiles, plate-like structures are used for obtaining lightweight structures. Rivet joints and spot welding are being used for bonding plates over many years, and adhesive bonding is being used in recent years [1], given its advantages. One advantage is the high joint stiffness. In adhesive bonds, the bond is generally distributed over a wider area when compared to other bonding techniques, and thus, joint stiffness increases and this leads to lightweight structures. Another advantage involves the applicability to dissimilar materials such as steel - aluminum plates and aluminum - CFRP (carbon fiber reinforced plastic) plates [2]. However, adhesive areas require appropriate inspection and evaluation to maintain their quality and strength. For example, large plate parts are often curved and slightly distorted. Additionally, when the curved plates are attached with adhesives using robot arms in an automated assembly line, adhesive disbonds occasionally appear and lead to lower stiffness of the complete structure below the designed values. In order to avoid this type of adhesion failure, various types of nondestructive and destructive testing techniques are used at each manufacturing site.

Radiography testing (RT) and ultrasonic testing (UT) are widely used for nondestructive evaluation (NDE) and nondestructive testing (NDT) for the internal states of materials. Although RT provides images of internal states, it is typically not preferred for a production site because it requires shielding equipment owing to harmful radiation. Furthermore, RT involves measuring the penetration power of radiation, and therefore, 
Journal of Nondestructive Evaluation, Diagnostics and Prognostics of Engineering Systems

it requires access to both sides of the inspected area. Given that plate-structures in aircraft and automobiles include numerous adhesive areas that are accessible only from a single side, this requirement in RT is a crucial constraint. Conversely, UT is an unharmful technique that can be easily introduced in production sites. The most simple and reliable technique in UT involves an ultrasonic pulse echo technique [3] in which an ultrasonic pulse at a center frequency in the order of $\mathrm{MHz}$ is incident on the object requiring inspection, and material constants, delaminations, and defects along the ultrasonic path are evaluated from the echo signals. However, with respect to a thin plate below approximately $1 \mathrm{~mm}$, it is necessary to use a higher frequency range to separate the echoes from surfaces and defects, and this typically results in various difficulties in measurements. Therefore, Allin et. al developed a practical evaluation technique for adhesive bonds in automobile shell bodies using a dry-contact dabber probe that operates in the low-frequency range below $500 \mathrm{kHz}$ [4]. These findings are applied to later-developed ultrasonic bond testers. A through-transmission method that uses air-coupled ultrasonic transducers was developed as a noncontact evaluation technique and is a very useful technique when transducers are placed on both sides of the inspection area $[5,6]$.

Although several studies developed methods for evaluating adhesive bonds of thin plates, as described above, for use in practical inspections, users are demanding new evaluation techniques to obtain better adhesion quality and facilitate easy inspection. Therefore, the present study investigates adhesive bond imaging by noncontact measurements with single-side access especially for adhesive joints in car 
Journal of Nondestructive Evaluation, Diagnostics and Prognostics of Engineering Systems

shell bodies. In the study, a laser was used to generate and detect elastic waves in a noncontact manner. The noncontact ultrasonic NDE with laser is well known as laser ultrasonics [7-9]. In laser ultrasonics, C-scan images are generally obtained by pulse echo measurements. However, the inspected objects in this study are thin plates, and thus, a scanning laser source (SLS) technique is applied. Hayashi et. al proved that defect images could be obtained using the SLS technique [10-15]. SLS is a measurement technique for multiple waveforms generated at many points by a scanning laser and detected by a receiving device at a fixed position. Complete noncontact measurements with single-sided access are feasible by using a laser Doppler vibrometer (LDV) as a receiving device, and thus, this measurement technique solves most issues discussed above. In previous studies $[12,13]$, clear images of notch-type defects in a flat plate were obtained from frequency spectrum peaks for narrowband burst waves at multiple frequency components. Additionally, inner-wall thinning in a pipe was visualized using the SLS imaging technique [15].

A previous study discussed the applicability of the imaging technique for adhesive bonds and delaminations in thin plates using numerical analyses with a semianalytical finite element method [16]. The calculation results indicated that the energy of flexural waves generated by the laser source located at the adhesive area is lower than that at the other single-plate areas and is also significantly high when the laser source is located at the boundaries of an adhesive area and a nonadhesive area.

The present study discusses experimental applications of the imaging technique for NDE of adhesive bonding. In order to prove that the imaging technique can be 
Journal of Nondestructive Evaluation, Diagnostics and Prognostics of Engineering Systems

applied effectively to NDE of adhesive bonding, adhesive bonds in test plate specimens are visualized by the imaging technique in the same manner as our previous studies on defect imaging [10-15], and then the new imaging technique using broadband chirp wave is developed to improve their images. The next section describes the experimental setup used in the study. In the third section, imaging of adhesive bonds using frequency spectrum peaks for narrowband burst waves is described. In section 4 , adhesive bond imaging using broadband chirp wave is discussed. In section 5, the application of the imaging technique to adhesive bonds in a complex plate structure that consists of a tophat section and a flat plate that simulates the adhesive bonding of automotive bodies is described.

\section{EXPERIMENTAL SETUP}

The imaging technique with SLS uses the characteristic wherein energy of the flexural vibration varies with the apparent local bending stiffness at the laser source. Therefore, an experimental system for imaging requires laser equipment to generate flexural vibrations, Galvano mirror scanners for moving a laser spot, and an LDV to detect flexural vibration.

Figure 1 shows the experimental setup used in the experiments. This study used fiber laser equipment (SPI laser, G4 50W-S-HS) that controls the laser output by providing external modulation signals and thereby generates flexural vibrations with various waveforms [14]. A digital-analog converter (NI, USB-6343) was used to generate the external modulation signals. Electric signals to control the Galvano mirror scanner 
Journal of Nondestructive Evaluation, Diagnostics and Prognostics of Engineering Systems

were also output from the same DA converter. The flexural vibrations generated by the laser radiation that propagated along a plate were detected by the LDV (Polytec OFV5000). The received signals were amplified at $40 \mathrm{~dB}$ and recorded by a computer after they were digitized by an analog-digital converter (NI, PXI-5124). For each waveform recorded in the PC memory, bandpass filtering, calculations of frequency spectrum peaks, and numerical integrations of the frequency spectrum were performed in a Labview-based software created by the authors, and the values for each laser irradiation were instantly acquired.

In the next section, imaging experiments for an adhesive area are implemented by using narrowband burst waves that were also used in defect imaging experiments in previous studies [12-16]. Figure 2 (a) shows a schematic figure of the modulation signals used in the experiments in the next section. Rectangular burst waves at three different frequencies, namely $30 \mathrm{kHz}, 35 \mathrm{kHz}$, and $40 \mathrm{kHz}$, with durations of $3.3 \mathrm{~ms}$ each are connected in series. In section 4, a chirp wave with a frequency that varies linearly from $30 \mathrm{kHz}$ to $40 \mathrm{kHz}$ with a duration of $10 \mathrm{~ms}$ as shown in Fig. 2 (b) is used for the imaging experiments.

\section{IMAGING EXPERIMENTS FOR ADHESIVE BONDS USING NARROWBAND BURST WAVES}

Extant studies by Hayashi et. al provided images of defects on the back surface

of a plate and pipe using frequency spectrum peaks of narrowband burst waves [12-14]. The use of narrowband burst waves improves the signal to noise ratio in the frequency domain and suppresses the maximum laser energy. This is advantageous for NDE as it is 
Journal of Nondestructive Evaluation, Diagnostics and Prognostics of Engineering Systems

possible to increase the total energy of the laser output while avoiding surface damages.

Here, we generated flexural waves at $30 \mathrm{kHz}, 35 \mathrm{kHz}$, and $40 \mathrm{kHz}$ using modulation signals as shown in Fig. 2 (a).

Figure 3 shows a schematic of the test plate used in the experiments. Two aluminum alloy plates measuring $200 \mathrm{~mm} \times 300 \mathrm{~mm} \times 1 \mathrm{~mm}$ were attached with strong double-faced tapes that retain their shape after bonding. The dark gray areas in Fig. 3 correspond to the bonding regions. A nonbonding gap of $50 \mathrm{~mm}$ was created between the bonded regions to model an adhesion failure. The receiving point is located at the right center of the surface plate. A laser beam for generating waves was rastered over the rectangular area enclosed by dashed lines at $1.0 \mathrm{~mm}$ increments and at $50 \mathrm{~ms}$ time intervals. The area measures $40 \mathrm{~mm} \times 100 \mathrm{~mm}$, and thus, the measurement time for 41 $\times 101$ points, without averaging, approximately corresponded to $41 \times 101 \times 50 \mathrm{~ms}=3.5$ min. The received signals were recorded from the commencement of laser irradiation for $12 \mathrm{~ms}$. All waveforms were filtered in the software using a bandpass filter with cutoff frequencies of $10 \mathrm{kHz}$ and $60 \mathrm{kHz}$. Figure 4 shows one of the received signals and its frequency spectrum. The peaks appearing at $21 \mathrm{kHz}$ and their multiples in the Fourier spectrum (b) correspond to intrinsic noise that originates from the experimental system. Figure 5 (a), (b), and (c) show images plotted using the peak values at $30 \mathrm{kHz}, 35 \mathrm{kHz}$, and $40 \mathrm{kHz}$, respectively. The peak values plotted in an image were normalized by the maximum value in the image. The images are shown in gray scale where the maximum values are indicated in black and zero values are indicated in white. The figures depict the resonant patterns that vary with frequencies, and the bonded regions that are 
Journal of Nondestructive Evaluation, Diagnostics and Prognostics of Engineering Systems

supposed to be located at the area enclosed by dashed lines did not appear clearly. In

Figure $5(d)$, the averaged image of images $(a)-(c)$ provides a clearer image wherein the resonant patterns are reduced and adhesive areas are slightly brighter when compared to the others. The averaging procedure was presented in a previous study to enhance defect images $[12,15]$, and this is termed as frequency image averaging (FIA). The result indicates that the FIA is useful for imaging adhesive bonds. Additionally, the results indicate that smaller values were obtained at the adhesive bonds, and this agrees well with the numerical analyses obtained in our previous study [16].

\section{IMAGING EXPERIMENTS USING A BROADBAND CHIRP WAVE}

Following previous studies, FIA using narrowband burst waves was applied to create an image of adhesive bonds in the previous section. However, it still contains resonance patterns, and the adhesive bonds were not clearly visualized. We infer that the use of more frequency components reduces the resonance patterns more efficiently. If a high number of frequency components are involved, then the modulation signals correspond to a chirp wave as shown in Fig. 2 (b). Therefore, the imaging experiments discussed in this section were conducted using a rectangular chirp wave as a modulation signal.

In order to avoid noise peaks at $21 \mathrm{kHz}$ and $42 \mathrm{kHz}$, chirp modulation signals that linearly vary from $30 \mathrm{kHz}$ to $40 \mathrm{kHz}$ with a duration of $10 \mathrm{~ms}$ are used as shown in Fig. 2 (b). Figure 6 shows a waveform that is detected for the chirp modulation signals and its frequency spectrum. The frequency spectrum exhibits slightly higher intensity in the 
Journal of Nondestructive Evaluation, Diagnostics and Prognostics of Engineering Systems

range from $30 \mathrm{kHz}$ to $40 \mathrm{kHz}$, and this indicates that elastic waves are appropriately generated in the desired frequency range. To utilize all frequency components in the range, square values of the frequency spectrum, as shown in Fig. 6 (b), were numerically integrated from $30 \mathrm{kHz}$ to $40 \mathrm{kHz}$, and the integration values are plotted in gray scale.

The square of the frequency spectrum is generally used as an index of signal energy in the field of acoustic emission. Integration of the square of the frequency spectrum in a whole frequency range is described by the integration of signals in the time domain using Parseval's equality as follows:

$$
\int_{-\infty}^{\infty}|x(t)|^{2} d t=\int_{-\infty}^{\infty}|X(2 \pi f)|^{2} d f
$$

where $t$ and $f$ denote time and frequency, respectively, $x(t)$ denotes the signal in the time domain, and $X(2 \pi f)$ denotes a Fourier transform of $x(t)$. While using $x(t)$, the Fourier transform is not required in a computer, and this results in slightly shorter calculation time. However, square values of the Fourier spectrum $X(2 \pi f)$ were integrated from $30 \mathrm{kHz}$ to $40 \mathrm{kHz}$ in the study because the detected waveforms contained very high noise at $21 \mathrm{kHz}$ and $42 \mathrm{kHz}$. Figure 7 shows the image where the values are plotted. As shown in Fig. 5, the maximum and minimum values are denoted as black and white, respectively. When compared with Fig. 5 in which narrowband burst waves at three frequencies are used, the resonance patterns are significantly reduced and adhesive bonds are clearly visualized in Fig. 7. If wider frequency range can be used to create an image, spurious images would be reduced more significantly. 
Journal of Nondestructive Evaluation, Diagnostics and Prognostics of Engineering Systems

As described in sections 3 and 4 , the test specimen consists of two flat plates with strong double-faced tapes for fundamental experiments of SLS imaging. The imaging experiments described in this section were conducted using a complex plate-structure wherein a top-hat section and a flat plate are bonded with adhesives to simulate the structure of automotive shell bodies.

Figure 8 shows a schematic of the test plate-structure with adhesive bonds that depicts its dimensions, rastering areas, and a receiving point. A two-part epoxy adhesive was applied in bands with a width of $20 \mathrm{~mm}$. One of the bands was separated by a gap of approximately $19 \mathrm{~mm}$. The gap is equivalent to the size of adhesive failure in automotive bodies. Rastering areas were located at two areas corresponding to $40 \mathrm{~mm}$ $\times 100 \mathrm{~mm}$ each that are enclosed by dashed lines as shown in the figure. Considering an unfavorable condition that we cannot place a receiving point at the same level of the rastering areas, the receiving point was located on top of the hat.

The laser beam was incident for $10 \mathrm{~ms}$ at each rastering point, and the waveforms were recorded for $12 \mathrm{~ms}$ after the laser irradiation commenced. The laser beam was rastered at $1.0 \mathrm{~mm}$ increments and at $50 \mathrm{~ms}$ time intervals as stated in sections 3 and 4. Thus, a period of approximately 3.5 min was involved in creating each image at the left and right areas. Figure 9 shows the images obtained using FIA of narrowband burst waves at three frequencies corresponding to $30 \mathrm{kHz}, 35 \mathrm{kHz}$, and 40 $\mathrm{kHz}$. The bonded regions as well as some resonant patterns in the left and right areas (a) and (b) were roughly visualized. Conversely, in Fig. 10, images were obtained using the chirp wave as described in the previous section. Specifically, (a) and (b) that correspond 
Journal of Nondestructive Evaluation, Diagnostics and Prognostics of Engineering Systems

to the bonded regions in the left and right areas, respectively, appear more distinct as resonance patterns were reduced. In both Figs. 9 and 10, dark lines were observed at the borders between bonded regions and nonadhesive regions. The border lines were observed in the numerical calculations in a previous study [16], and therefore, it is concluded that the experimental images correspond to reasonable results.

\section{CONCLUSIONS}

The study involved investigating the imaging of adhesive bonds using an SLS technique. First, FIA with narrowband burst waves was applied to the imaging of adhesive bonds, and the images of adhesive bonds as well as resonance patterns were visualized. Subsequently, the use of a chirp wave enhanced the images of the adhesive bonds and reduced spurious resonance patterns. Additionally, images of adhesive bonds were obtained even for a complex plate structure that consisted of a top-hat section and flat plate, and this demonstrates that the technique can be widely applied to various types of plate structures.

\section{FUNDING}

This study was supported by JSPS KAKENHI Grant Number 17H02052. 
Journal of Nondestructive Evaluation, Diagnostics and Prognostics of Engineering Systems

\section{REFERENCES}

[1] Barnes, T.A., and Pashby, I.R., 2000, "Joining Techniques for Aluminium Spaceframes Used in Automobiles," J. Mater. Process. Technol., 99, pp. 62-71.

[2] Martinsen, K., Hu, S.J., and Carlson, B.E., 2015, "Joining of Dissimilar Materials," CIRP Ann. - Manuf. Technol., 64, pp. 679-699.

[3] Titov, S. A., , Maev, R. Gr. and Bogachenkov, A. N., 2008, "Pulse-Echo NDT of Adhesively Bonded Joints in Automotive Assemblies," Ultrasonics, 48, pp. 537-546. DOI: 10.1016/j.ultras.2008.07.001

[4] Allin, J. M., Cawley, P., and Lowe, M. J. S., 2003, “Adhesive Disbond Detection of Automotive Components Using First Mode Ultrasonic Resonance," NDT\&E International, 36, pp. 503-514. DOI: 10.1016/S0963-8695(03)00045-8

[5] Schindel, D.W., and Hutchins, D.A., 1995, "Through-Thickness Characterization of Solids by Wideband Air-Coupled Ultrasound," Ultrasonics, 33, pp. 11-17.

[6] Chimenti, D.E., 2014, "Review of Air-Coupled Ultrasonic Materials Characterization," Ultrasonics, 54, pp. 1804-1816.

[7] Tam, A. C., 1984, "Pulsed-Laser Generation of Ultrashort Acoustic Pulses: Application for Thin-Film Ultrasonic Measurements," Appl. Phy. Lett., 45, pp. 510-512.

[8] Scruby, C. B., and Drain, L. E., 1990, Laser Ultrasonics: Techniques and Applications, Adam Hliger, New York. ISBN 0-7503-0550-7

[9] Fomitchov, P. A., Kromin, A. K., Krishnaswamy, S., and Achembach, J. D., 2004, "Imaging of Damage in Sandwich Composite Structures Using a Scanning Laser Source Technique," Composites: Part B, 35, pp. 557-562. DOI:

10.1016/j.compositesb.2004.04.007

[10] Hayashi, T., Murase, M., and Salim, M. N., 2009, "Rapid Thickness Measurements Using Guided Wave from Scanning Laser Source," J. Acoust. Soc. Am., 126, pp. 11011106.

[11] Hayashi, T., Murase, M., and Kitayama, T., 2012, "Frequency Dependence of Images in Scanning Laser Source Technique For a Plate," Ultrasonics, 52, pp. 636-642.

[12] Hayashi, T., 2016, "Imaging Defects in a Plate with Complex Geometries," Appl. Phys. Lett., 108, 81901. 
Journal of Nondestructive Evaluation, Diagnostics and Prognostics of Engineering Systems

[13] Hayashi, T., 2017, "High-Speed Non-Contact Defect Imaging for a Plate-Like Structure," NDT E Int., 85, pp. 53-62.

[14] Hayashi, T., and K., Ishihara, 2017, "Generation of Narrowband Elastic Waves with a Fiber Laser And its Application to the Imaging of Defects in A Plate," Ultrasonics, 77, pp. 47-53.

[15] Hayashi, T., 2017, "Non-Contact Imaging of Pipe Thinning Using Elastic Guided Waves Generated and Detected by Lasers," Int. J. Press. Vessel. Pip., 153, pp. 26-31.

[16] Hayashi, T., and Nakao, S., 2017, "Energy Analyses for the Imaging Technique of Bonded Regions and Delaminations in a Thin Plate," Materials Transaction, 58(9), pp. 1264-1273. DOI: 10.2320/matertrans.M2017151 
Journal of Nondestructive Evaluation, Diagnostics and Prognostics of Engineering Systems

\section{Figure Captions List}

Fig. $1 \quad$ Experimental set-up

Fig. 2 Modulation signals for controlling laser output. (a) Rectangular burst waves used in the experiments in Sec. 3. (b) Chirp wave varies linearly from $30 \mathrm{kHz}$ to $40 \mathrm{kHz}$ as used in the experiments in Sec. 4. Period and pulse width of the rectangular waves are modified to schematically describe frequency change.

Fig. 3 Test plate used in the experiments

Fig. $4 \quad$ Typical waveform and its frequency spectrum while using narrowband burst waves at three frequencies. (a) Waveform. (b) Frequency spectrum.

Fig. 5 Images of the bonded region by SLS given the use of Frequency spectrum peaks of narrowband burst waves. (a) $30 \mathrm{kHz}$. (b) $35 \mathrm{kHz}$. (c) $40 \mathrm{kHz}$. (d) Average.

Fig. 6 Typical waveform and its frequency spectrum while using chirp modulation signals. (a) Waveform. (b) Frequency spectrum.

Fig. 7 Image of bonded regions by SLS given the use of chirp modulation signals

Fig. 8 Schematic figure of the test plate-structure with adhesive bonds

Fig.9 Images of bonded regions using FIA by narrowband burst waves. (a) Left area. (b) Right area. 
Journal of Nondestructive Evaluation, Diagnostics and Prognostics of Engineering Systems

Fig. 10 Images of bonded regions using chirp wave. (a) Left area. (b) Right area. 
Journal of Nondestructive Evaluation, Diagnostics and Prognostics of Engineering Systems

Fig. 1

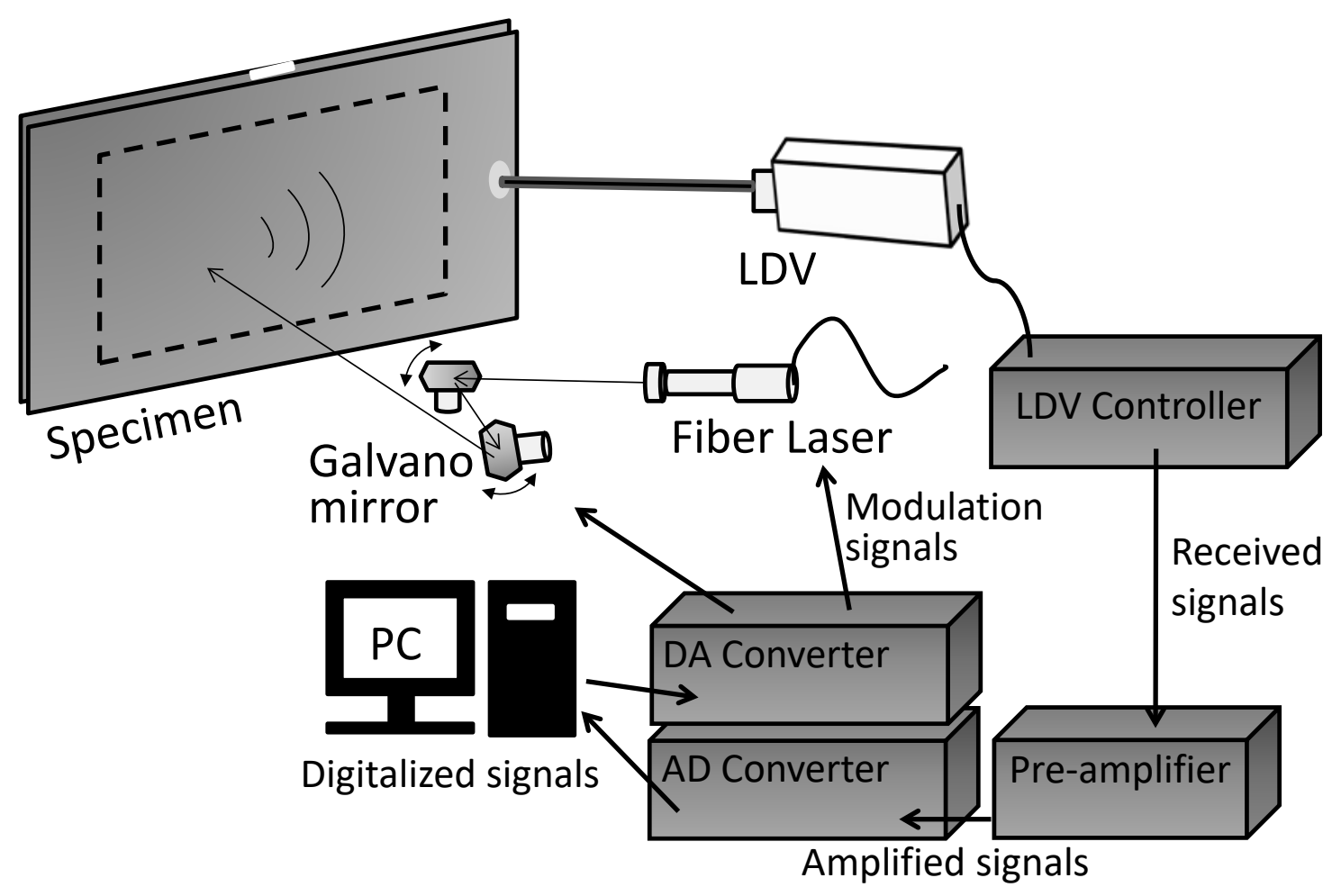


Journal of Nondestructive Evaluation, Diagnostics and Prognostics of Engineering Systems

Fig. 2

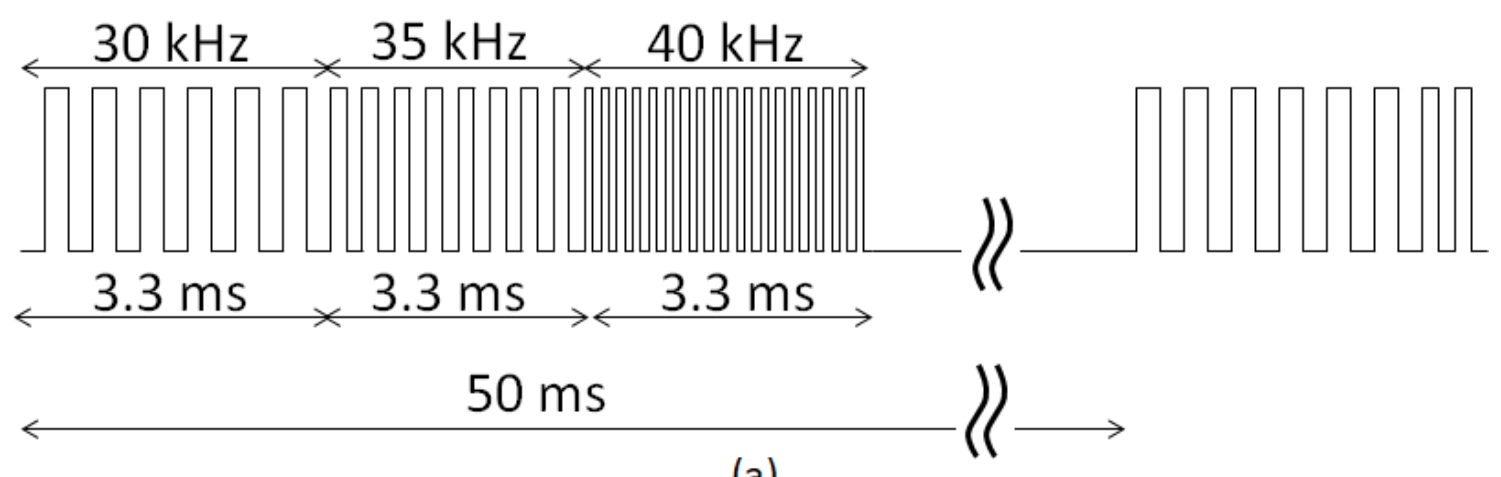

(a)

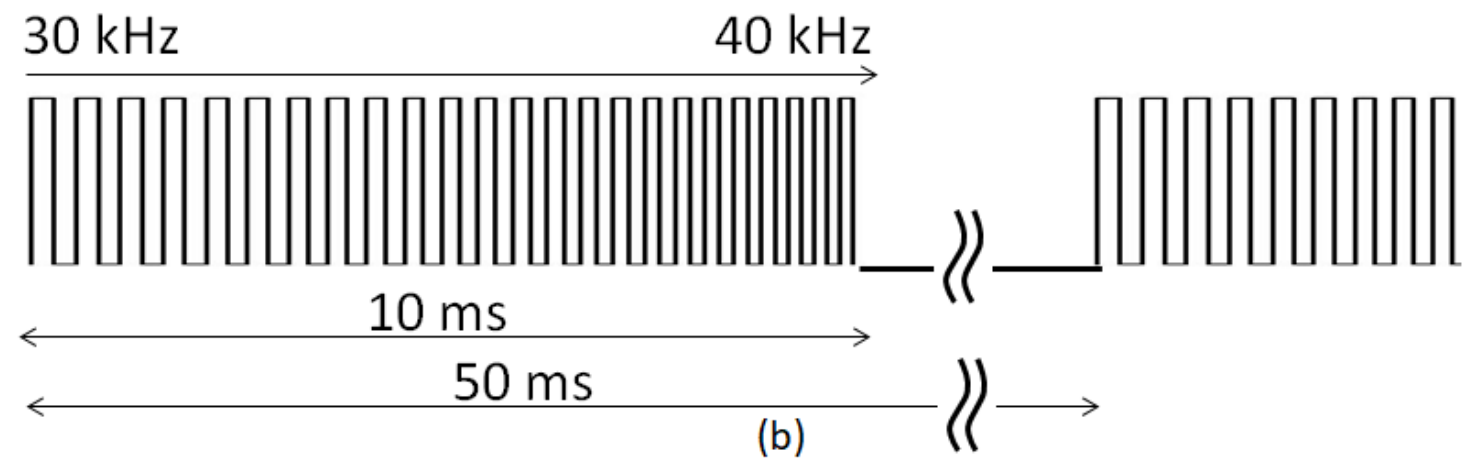


Journal of Nondestructive Evaluation, Diagnostics and Prognostics of Engineering Systems

Fig. 3

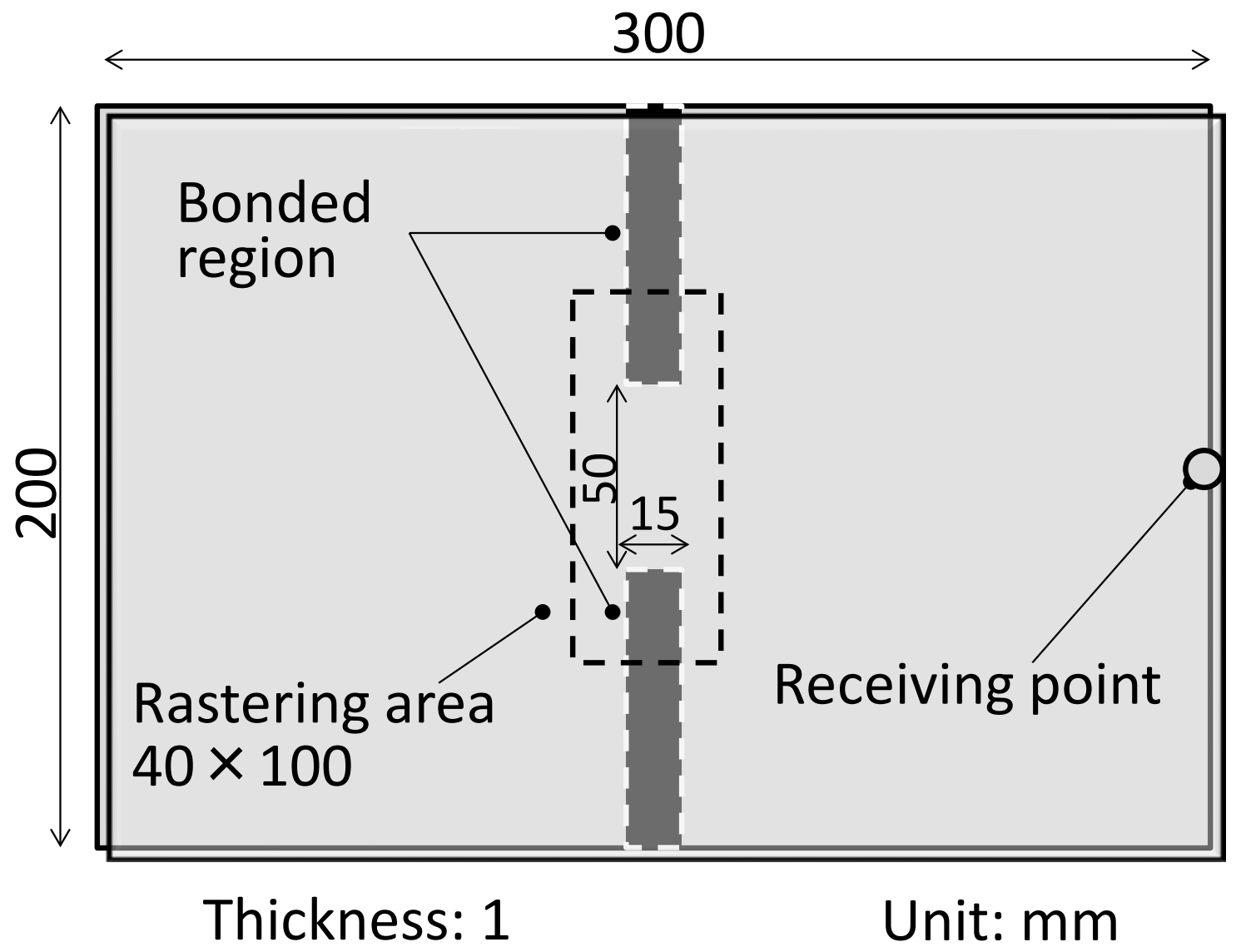


Journal of Nondestructive Evaluation, Diagnostics and Prognostics of Engineering Systems

Fig. 4

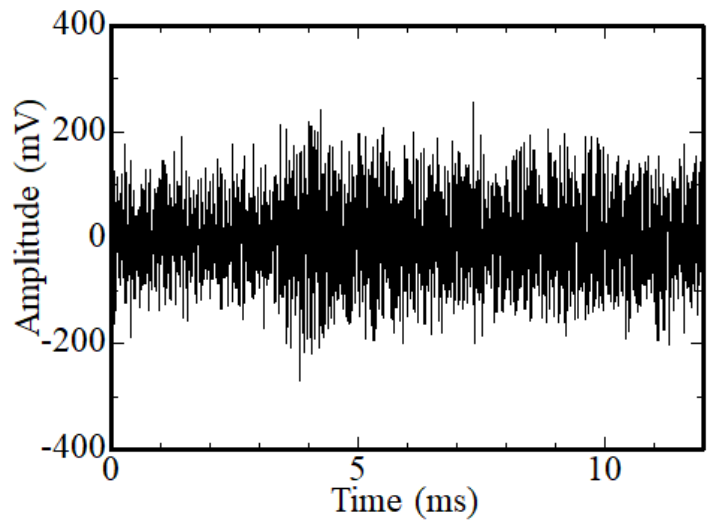

(a)

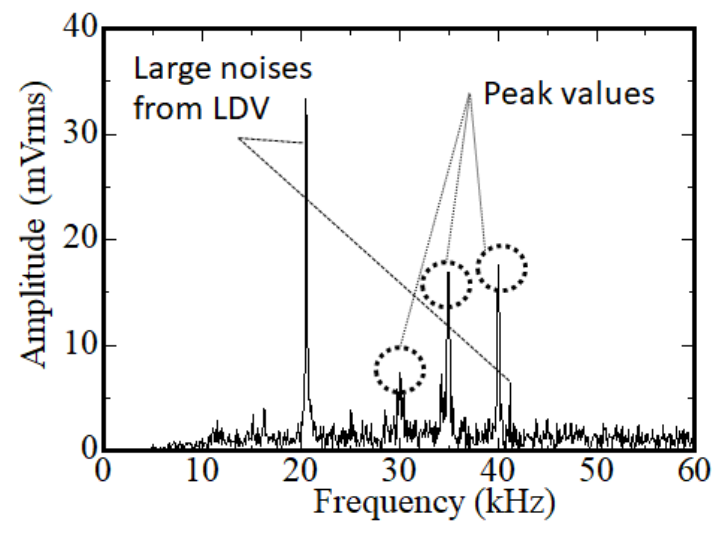

(b) 
Journal of Nondestructive Evaluation, Diagnostics and Prognostics of Engineering Systems

\section{Fig. 5}

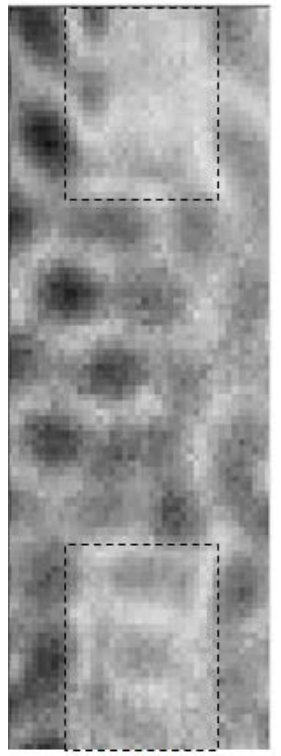

(a)

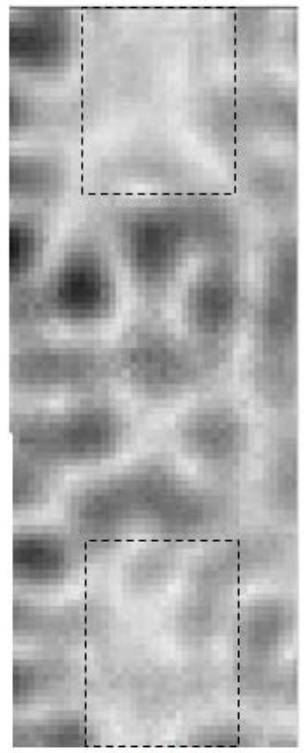

(b)

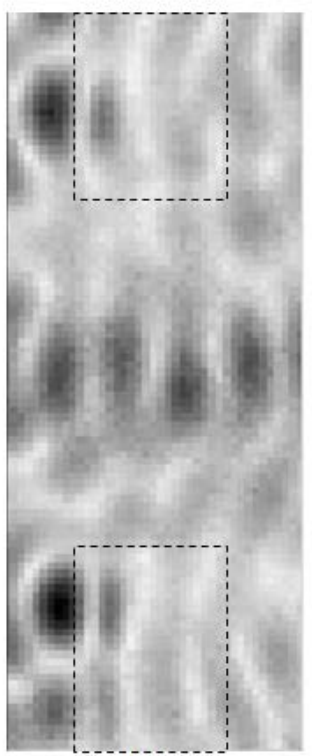

(c)

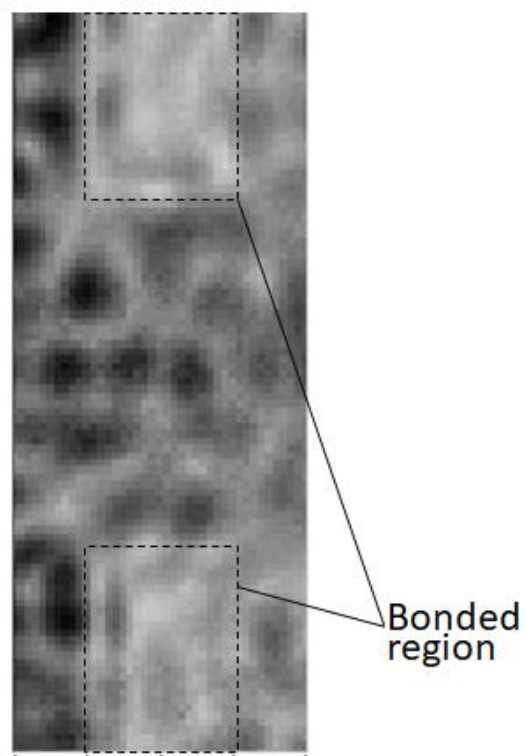

(d) 
Journal of Nondestructive Evaluation, Diagnostics and Prognostics of Engineering Systems

Fig. 6

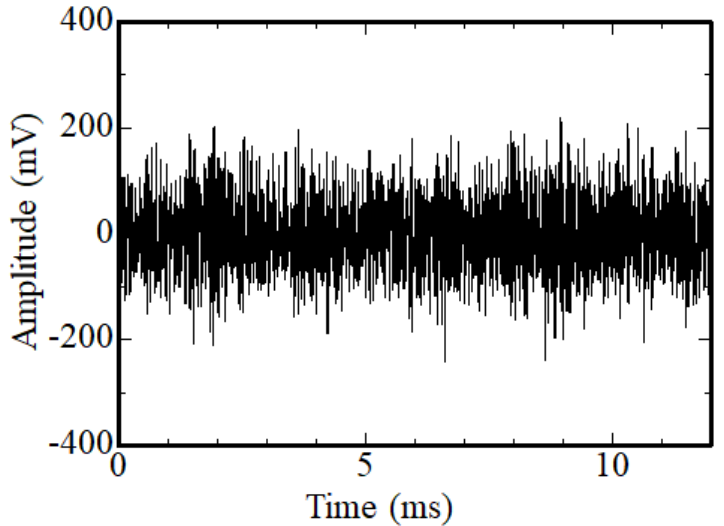

(a)

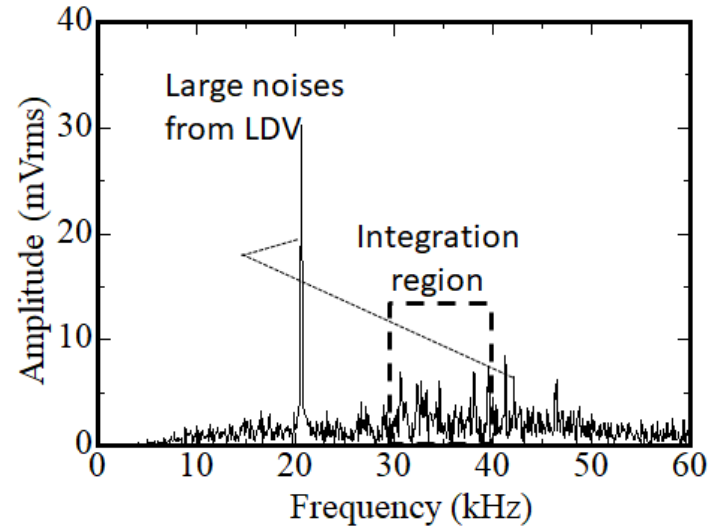

(b) 
Journal of Nondestructive Evaluation, Diagnostics and Prognostics of Engineering Systems

Fig. 7

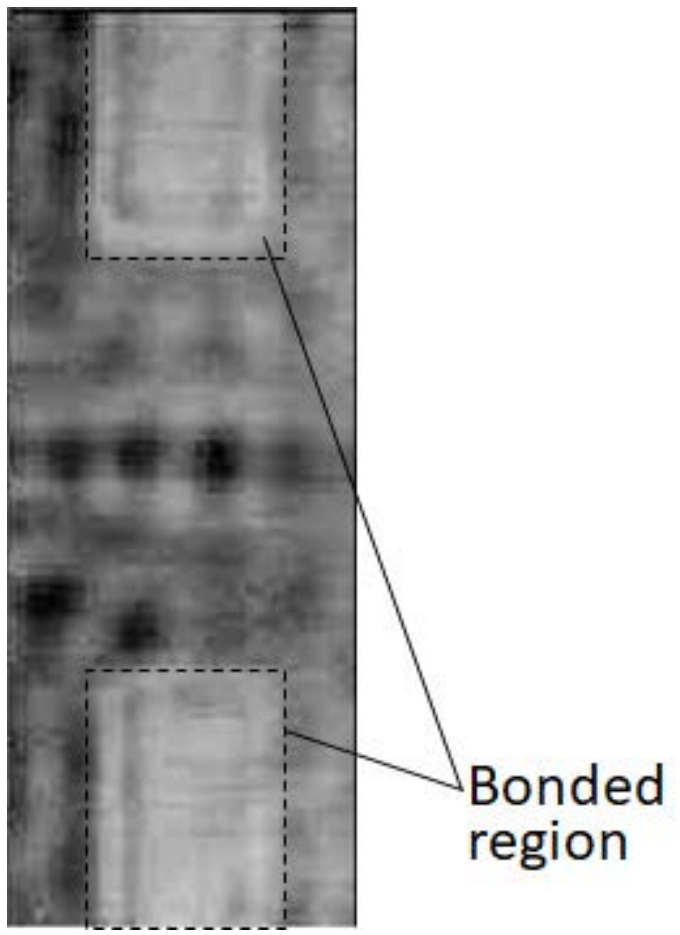


Journal of Nondestructive Evaluation, Diagnostics and Prognostics of Engineering Systems

Fig. 8

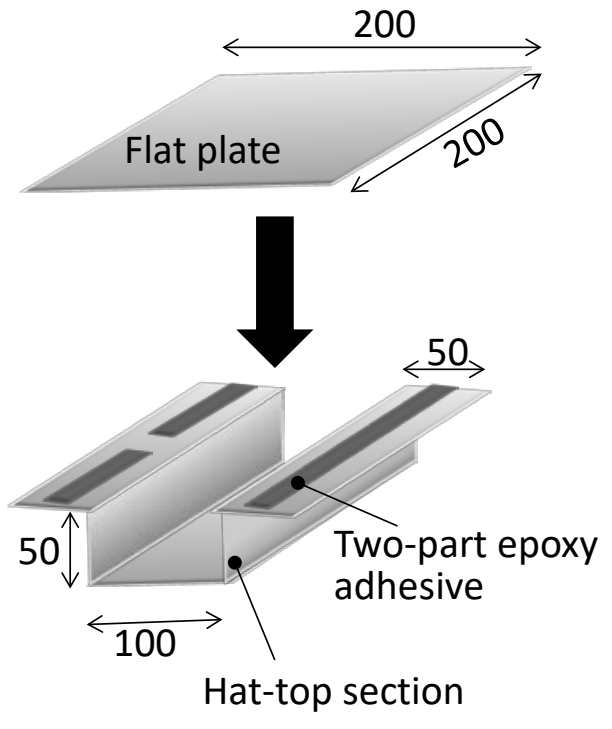

Unit: $\mathrm{mm}$

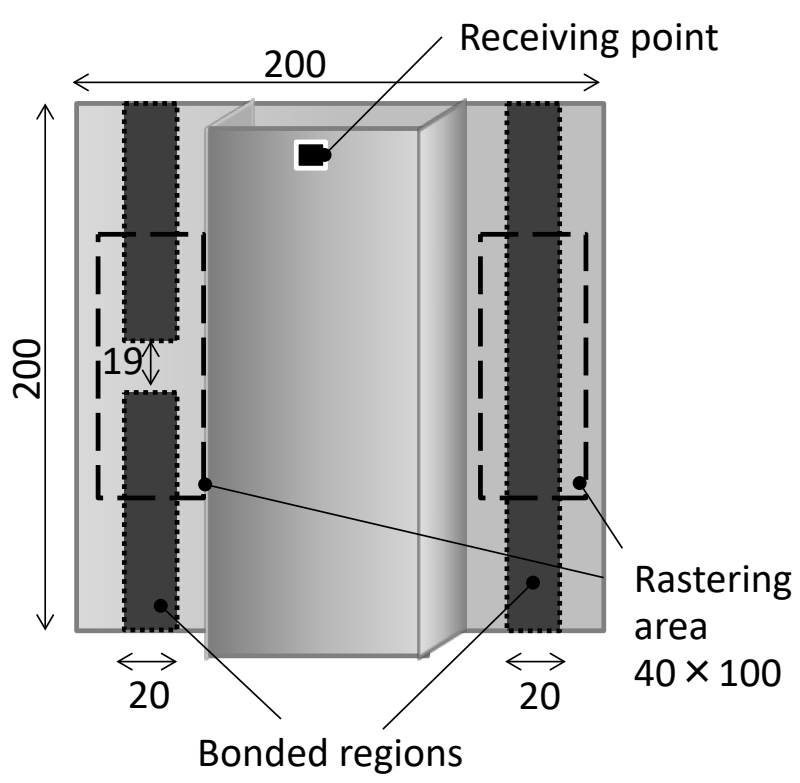


Journal of Nondestructive Evaluation, Diagnostics and Prognostics of Engineering Systems

Fig. 9

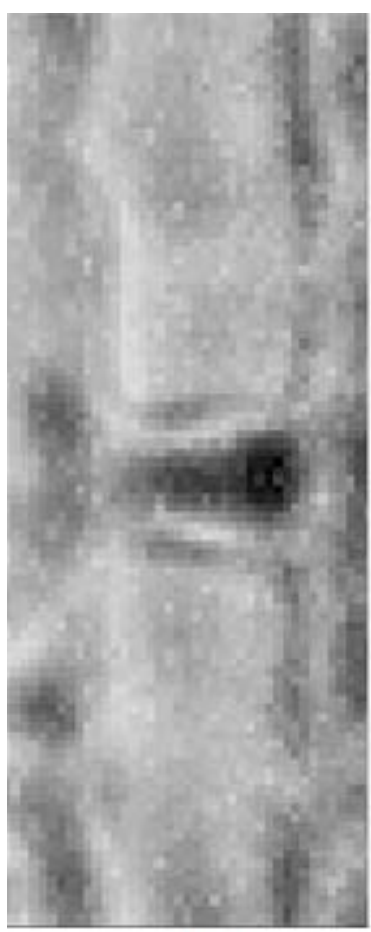

(a)

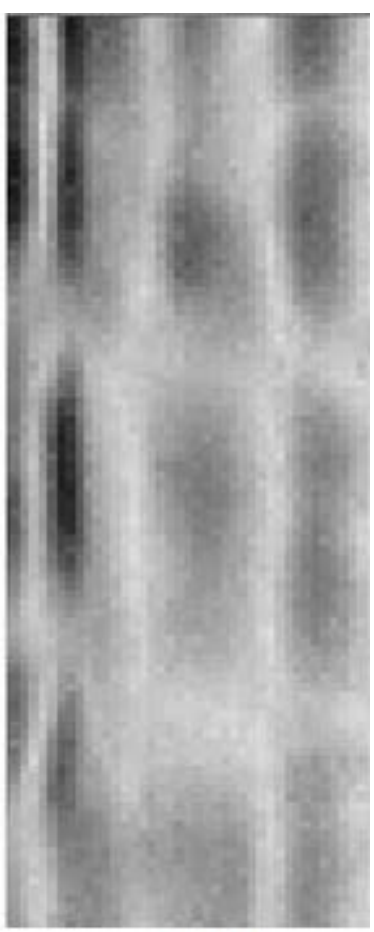

(b) 
Journal of Nondestructive Evaluation, Diagnostics and Prognostics of Engineering Systems

Fig. 10

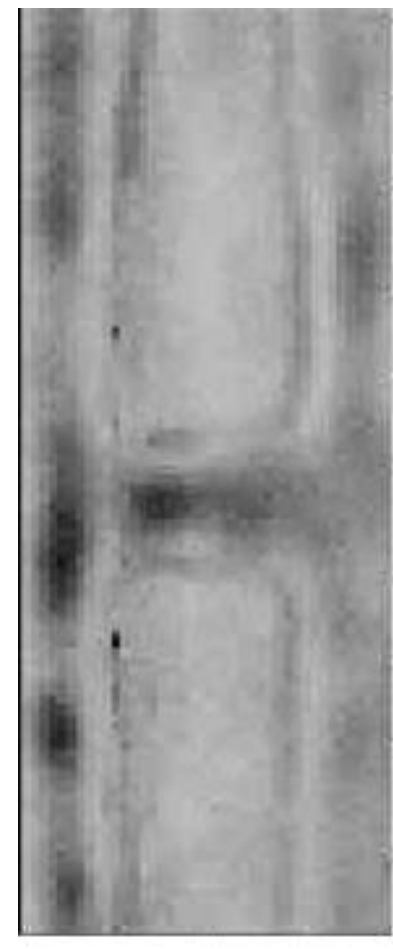

(a)

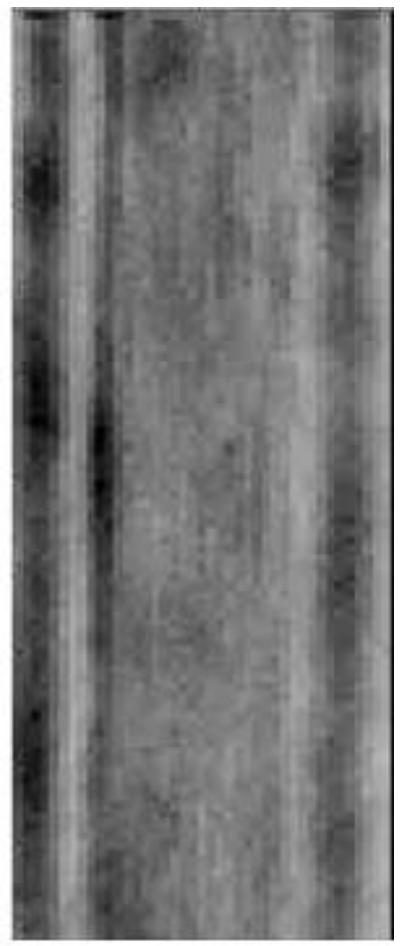

(b) 